\title{
¿PSICOANÁLISIS \\ CON LA COMUNIDAD? DEL EFECTO \\ DE LO GRUPAL AL LAZO SOCIAL ASISTIDO POR OTROS
}

\section{Cecilia Vaca H.}

Recibido: 29 de marzo de marzo de 2016

Aprobado: 6 de junio de 2016

*Pontificia Universidad Católica del Ecuador, Facultad de Psicología, Quito, Ecuador (cvaca128@puce.edu.ec). 



\section{¿PSICOANÁLISIS \\ CON LA COMUNIDAD? DEL EFECTO \\ DE LO GRUPAL AL LAZO SOCIAL ASISTIDO POR OTROS}

Cecilia Vaca H.

Palabras clave: producción de saber, lazo social, psicoanálisis aplicado, práctica entre varios, psicoanálisis con la comunidad.

Key words: wisdom production, social link: applied psychoanalysis, practice among several, psychoanalysis with community.

\section{RESUMEN}

Este artículo proviene de interrogantes formuladas durante varios años de experiencia clínica en contextos institucionales públicos que se ocupan de problemáticas complejas como la violencia intrafamiliar o el maltrato infantil. Por tanto, este estudio se fundamenta en una clínica psicoanalítica en condiciones distintas al de su desenvolvimiento en la consulta privada y en el dispositivo de la cura tipo. Así, el artículo recoge el aporte de diversos autores cuya clínica se ha desarrollado en ese sentido, aunque a través del trabajo con algunas problemáticas diferentes. Al mismo tiempo, distingue este tipo de dispositivos respecto de aquellos que trabajan con y desde una perspectiva grupal. Se propone una revisión teórico-clínica de dispositivos psicoanalíticos poco difundidos en nuestro contexto, los mismos que plantean elementos y operaciones que, a nuestro modo de ver, establecen la condición de posibilidad de un trabajo psicoanalítico con la comunidad. 


\begin{abstract}
This article is motivated by a number of questions and issues that appeared during several years of clinical experience at public institutions that deal with complex issues such as domestic violence or child abuse. Consequently, this paper aims to discuss different approaches to psychoanalytical practice compared to ones applied on traditional private practice. This investigation collects a number of approaches from various authors whose clinical
\end{abstract}

work has been developed in institutional contexts. At the same time, the article distinguishes these approaches from those that work with and from a group perspective. This paper presents a theoretical and clinical review of psychoanalytic devices which have not been widespread in our context. These devices present the elements and operations which, in our view, would set the conditions for a psychoanalytical work with communities.

\section{INTRODUCCIÓN}

Las inquietudes y preguntas que llevan a la formulación de este artículo surgen en el contexto de varios años de ejercicio de la clínica en servicios de atención públicos especializados, cuya labor se desarrolla a través de la intervención de equipos multi o interdisciplinarios. Algunos vectores confluyen en su planteamiento.

En primer lugar, las dificultades inherentes a las problemáticas que constituyen la razón de ser de estos servicios: situaciones de maltrato, violencia, vulneraciones graves de derechos, así como el desbordamiento de la capacidad efectiva de acción de estos debido a su saturación y, muchas veces, a la escasez de recursos y de personal.
Ante este panorama, se ha impuesto un prejuicio generalizado acerca de las posibilidades de atención psicológica para la población que la solicita: se requiere de intervenciones rápidas, eficientes y poco costosas (Cfr. Laurent, 2000); por tanto, el psicoanálisis -entendido en la versión del dispositivo tradicional- aparece como la antítesis de este requerimiento pues toma demasiado tiempo, no trabaja en función de la adaptación y resulta costoso.

Además, el consenso establecido a nivel de los servicios públicos y de ONGs que intervienen en problemáticas psicosociales respecto a que la complejidad de las situaciones aten- 
didas requiere de un abordaje interdisciplinario, y las dificultades que este abordaje plantea al tratarse de un ideal que difícilmente llega a consumarse, imprime un modo de hacer en el cual confluyen, de modo conflictivo, los saberes de distintas disciplinas: derecho, psicología, medicina, trabajo social, pedagogía y otras. Muchas de las veces, en el conflicto por la primacía de la perspectiva de cada una de las disciplinas, o ante la pasividad que otorga a un solo discurso-práctica la predominancia de la intervención, se pierde la posibilidad de realizar intervenciones certeras. Así, resultan numerosas las situaciones en las cuales las problemáticas se repiten, persisten aún después de la intervención, o aparecen nuevos síntomas que agudizan el pedido de los usuarios. De modo tal que la efectividad anhelada, la rapidez y el menor costo se presentan más como ilusiones, a veces difíciles de admitir, que como efectos duraderos en la realidad.

Ahora bien, si del lado de las políticas públicas e internacionales se insiste en el discurso de la eficacia y la efectividad, del lado de la producción psicoanalítica se ha insistido, al menos en el contexto ecuatoriano, en el trabajo clásico del psicoanalista con su paciente, un dispositivo que propone y justifica un procedimiento de larga duración y que, sin ilusiones, ofrece poner en relación al sujeto con su deseo. Dado este planteamiento, su posibilidad de acción, en este contexto, resulta alcanzable para un sector restringido de la población: aquel que dispone de un cierto capital cultural y está en posibilidades de asumir los costos y el tiempo que este trabajo requiere. El trabajo psicoanalítico en institución es todavía poco difundido, poco sistematizado y fundamentalmente poco visibilizado.

La pregunta por la posibilidad de operativizar los conceptos psicoanalíticos de un modo distinto al del tratamiento clásico, y la interrogación sobre las posibilidades de encontrar otros modos y condiciones para la apuesta por el sujeto y la escucha de la dimensión escribir de lo inconsciente, en medio de problemáticas complicadas por la diversidad de factores, conflictos y políticas que confluyen en su aparecimiento, han guiado nuestra búsqueda profesional por cerca de dos décadas. Las ideas que se exponen a continuación proponen algunos hallazgos. 


\section{EL EFECTO DE LO GRUPAL: PSICOANÁLISIS DE GRUPOS Y FRAGMENTACIÓN DE LA INSTITUCIONALIDAD PSICOANALÍTICA}

"Encuentro ahí la impresión del milagro de los primeros freudianos: encontrar la fuerza viva de la intervención en el mismo callejón sin salida de una situación (Lacan, 1947, p. 4)".'

El aporte fundamental e imprescindible que realiza el psicoanálisis a las ciencias humanas es el dar cuenta de un modo sistemático de la dimensión inconsciente constituyente de lo humano. Desde ese aporte, el psicoanálisis se ha consagrado como la ciencia², o el campo, del inconsciente por excelencia.

1 "La psiquiatría inglesa y la guerra" se trata de un artículo aparecido en la revista L'Evolution psychiatrique, 1947, vol. 1. Vuelto a publicar en AA.W., La querelle des diagnostics. Paris: Navarin, 1986, pp. 15-42. La versión consultada fue traducida por Hernán Scholten.

${ }^{2}$ Hay una profunda discusión respecto del estatuto científico del psicoanálisis impulsada desde el interior de su movimiento. Así, algunos psicoanalistas lo postulan como discurso o como campo (Allouch, 1994), como saber diferenciándolo de la empresa científica. Lacan se mostraba crítico respecto al discurso y la práctica científica y proponía que el psicoanálisis daría cuenta precisamente de aquello que la ciencia excluye. Sin embargo, hay también psicoanalistas que reivindican al psicoanálisis como una ciencia, la única ciencia capaz de dar cuenta de lo Inconsciente (Cfr. Braunstein, 2003).
A más de esto, entre las instituciones psicoanalíticas existe la idea extendida que el dispositivo de la cura tipo, creado por Freud a finales del siglo XIX, es concomitante a la capacidad de dar cuenta de esta dimensión inconsciente y de la posibilidad de generar cambios en la subjetividad del paciente a partir de su accionar. La generalización de esta idea, y el despliegue de alternativas a la misma, han escindido en varias ocasiones al movimiento psicoanalítico; de modo que la fragmentación de las instituciones y la proliferación de las prácticas son ya una característica de la historia del psicoanálisis.

Un ejemplo interesante de esta proliferación de modalidades de trabajo generador de rupturas lo constituye el psicoanálisis de grupos. Se trata de una modalidad que ejemplifica el nivel conflictivo y de disputa puesto en marcha a partir de la instauración de modalidades de trabajo psicoanalítico diferentes al propuesto por Freud.

Luego de la segunda guerra mundial surgieron iniciativas que planteaban modalidades de intervención grupales como las desarrolladas por Bion y Rickman (Gordon, Bain y Gould, 1996) en Inglaterra o las de Anzieu y Martin 
(1971) y Käes (1997) en Francia; al igual que otros dispositivos en Latinoamérica donde, por ejemplo, el trabajo de Pichón Riviere, inicialmente sustentado en el psicoanálisis, permitió el despliegue de todo un modelo de psicología social (Buzzaqui, 1998).

Sin embargo, esta proliferación del trabajo "psicoanalítico" grupal no ha sido aceptada por algunos sectores del psicoanálisis que ven en el mismo una claudicación al método, entendiéndolo como el modo de operar del dispositivo de la cura. De acuerdo a Buzzaqui (1998), algunos psicoanalistas acuden a los textos de Freud para afianzar una posición que deslegitima el trabajo psicoanalítico grupal, sustentándose en enunciados que proponen para comprender el funcionamiento grupal: "su relación con 'estados' como la hipnosis, el enamoramiento, la neurosis y la masas" (Buzzaqui, 1998, p. 165). A decir de Freud (1992) las agrupaciones humanas no podrían prescindir de un funcionamiento de masa: "la masa se nos aparece como un renacimiento de la horda primordial. Así como el hombre primordial se conserva virtualmente en cada individuo, de igual modo la horda primordial se restablece a partir de una multitud cualquiera de seres humanos" (Freud, 1992, pp. 113 y 117). La dura crítica de Freud a la tesis de Trotter sobre el instinto gregario lleva a Buzzaqui a plantear como propuesta freudiana una suerte de imposibilidad de lo gregario destacando que "en los vínculos sociales (en la masa, en los grupos) es la relación con el líder el elemento fundamental y no las relaciones entre los iguales" (Buzzaqui, 1998, p. 165).

También Lacan se refirió en la década de los años 1970 de un modo crítico a los planteamientos del psicoanálisis de grupos. En el Atolondradicho de 1973 lo cuestiona, formulando que el efecto de grupo se sitúa a nivel de lo imaginario: "sopeso el efecto de grupo según lo que añade de obscenidad imaginaria al efecto de discurso" (Lacan, 1973, p. 15; cit. en Buzzaqui 1998, p.165). Y en otros textos verterá afirmaciones como: "Al contrario, el creciente desarrollo, en este siglo, de los medios para actuar sobre el psiquismo una manipulación concertada de las imágenes y de las pasiones, de las que ya se ha hecho uso con éxito contra nuestro juicio, nuestra firmeza y nuestra unidad moral, darán lugar a nuevos abusos de poder" (Lacan; cit. en Buzzaqui 1998, p.169). El efecto de grupo fijaría y reforzaría la función de desconocimiento propia de lo imaginario, y el grupo se constituiría, debido a esos efectos miméticos y alienantes, en ese mismo registro. "(...) Lacan nunca ha dicho nada que diera a entender que esto imaginario se pudiera simbolizar, que fuera el lugar de algo distinto de un 
aumento de alienación. Se pasa de una verdadera cuestión de principio rebelde a cualquier puesta a prueba. Eppur, si muove..." (Kaës, 1993, p. 87; cit. en Buzzaqui, 1998, p. 173).

Sin embargo, y según el devenir histórico diverso de las prácticas psicoanalíticas, el desarrollo de dispositivos de trabajo grupal y otros, también han encontrado en estos mismos autores, en la invitación freudiana para investigar la psicología de las masas (Freud, 1992, p.68) y en la postura lacanaina (Lacan, 1983, p.129) elementos para su desarroIlo. Esto se debe, quizás, a que el trabajo de estos pensadores no es un trabajo lineal y homogéneo sino que presenta posicionamientos distintos de acuerdo con el momento de su producción (incluido el momento histórico y el contexto social en el cual se realiza una formulación) articulados a las idas y vueltas de su propia elaboración teórica.

Buzzaqui llama la atención sobre un posicionamiento muy distinto de Lacan en la década del cuarenta cuando había finalizado la segunda guerra: "había elogiado la creatividad de los aportes ingleses, y sus diferentes facetas de intervención grupal como una práctica de posguerra". Por tanto, para Buzzaqui, Lacan en 1966 (Lacan 1983) y 1970 criticaba más el contexto socio ideológico en el cual aparecen las prácticas grupa- les: el de la psicología del Yo norteamericana, que promueve el concepto de un yo "autónomo" aliado de la cura, en detrimento del planteamiento freudiano. Y debido a esto no ejerció la misma crítica ante los trabajos de Bion y Rickman en la Inglaterra de posguerra. Se trataba pues de un contexto en el cual urgía la recomposición de los vínculos sociales. "Atento a los movimientos ideológicos y científicos, Lacan señala la cooperación de diversas disciplinas a partir de la gigantesca experiencia colectiva que significó la guerra" (Buzzaqui, 1998, p. 166-167).

Para Kaës, uno de los referentes más importantes del psicoanálisis grupal, la proposición lacaniana del "efecto de grupo" lanzada en el Atonlondradicho habría tenido "como efecto (de grupo) cerrar la investigación sobre el grupo para toda una corriente del psicoanálisis, al denunciar los efectos de grupo en lugar de proponerlos para el análisis" (Kaës, 1993, p. 86 cit. en Buzzaqui, 1998, p. 170). Los alcances que tuvo la crítica de Lacan a los efectos de grupo, no solamente cerraron la investigación sobre este tópico para el psicoanálisis lacaniano sino que han tenido repercusiones en la organización e institucionalidad psicoanalítica. Decía Lacan: "Lo mío parece una empresa desesperada (lo es por el hecho mismo, 
en el reside lo desesperado) porque es imposible que los psicoanalistas formen grupo. No obstante, el discurso psicoanalítico (es mi desbroce) puede precisamente fundar un vínculo social limpio de toda necesidad de grupo" (Lacan; cit. en Buzzaqui, 1998, p. 170-171; Cfr. Mannoni, 1992, pp. 108-110).

Situación que tal vez se vuelve reveladora cuando se considera la constante escisión en la organización psicoanalítica, la misma que es patente en el contexto ecuatoriano. Pareciera que precisamente por tratarse de un funcionamiento grupal, en el sentido lacaniano de efecto de grupo, lo que termina deshaciéndose es el vínculo. Sin embargo, aunque a través de este posicionamiento Lacan cancelaba las posibilidades de un trabajo de o sobre lo grupal, dejó explícita la diferencia entre grupo y vínculo social o lazo social.

Algunos psicoanalistas cercanos o seguidores de Lacan crearon posteriormente modalidades de trabajo que intervienen, a nuestro modo de ver, sobre el vínculo, sobre el lazo social, sin recurrir al efecto de grupo. De esta conflictividad psicoanalítica, respecto a los dispositivos de psicoanálisis grupal, retenemos precisamente el interrogante sobre las posibilidades de un trabajo que acoge el inconsciente sosteniéndose en algo del orden de lo colectivo o de lo plural y que puede prescindir del efecto de lo grupal o, en su defecto, crea las posibilidades de análisis de ese efecto.

Hacia fines del siglo $X X$, surgieron algunas modalidades de trabajo psicoanalítico que quizás no han sido tan difundidas como el psicoanálisis grupal y que, sin embargo, dan cuenta de cambios importantes en la manera de plantear y concebir la práctica psicoanalítica generando la posibilidad de hacer un trabajo que incluye la consideración del inconsciente de otra manera. Los dispositivos para la primera infancia instaurados por Doltó (2004) en Francia, la práctica entre varios de Di Ciacca (2011), como una de las modalidades del psicoanálisis aplicado, o la psicoterapia institucional de filiación psicoanalítica De Coninck, Oldenhove y otros (2014), dan cuenta de esta posibilidad. Es en este tipo de modalidades que se circunscribe el interés de este artículo y el de la práctica clínica desarrollada durante varios años.

A continuación se explican brevemente dos de estos dispositivos y algunas de las interrogantes que suscitan. Se trata de preguntas que van desde cuestiones metodológicas y operativas, hasta el estatuto epistemológico de la teoría que los produce y que estos dispositivos producen. 


\section{UN LAZO SOCIAL ASISTIDO POR OTROS}

Primeramente, y aunque el planteamiento del dispositivo psicoanalítico se diversificó con modalidades como el psicoanálisis de grupo, este continúa circunscrito a un modo de intervención definida en un proceso en el cual la distribución de funciones: psicoanalista-paciente o pacientes, el horario de encuentro, el lugar físico para el mismo, están predispuestos y constituyen parte de las condiciones que lo configuran.

A continuación se mencionarán brevemente algunos elementos que diferencian el trabajo de un dispositivo analítico de otros dispositivos terapéuticos. Estos elementos pueden agruparse en torno de aspectos tales como: la producción del saber, el uso del poder y la función de la transferencia. A diferencia de varios dispositivos afiliados a enfoques psicoterapéuticos que promueven la "adaptación" del sujeto a su medio y que, por tanto, implementarán una serie de procedimientos, acciones y tareas enfocadas a ese fin; de modo que quien detenta el saber es el psicoterapeuta y es a través de su saber que ejerce un poder sobre su paciente. En el dispositivo psicoanalítico, el saber depende de su develamiento por parte del analista desde la producción del paciente; el psicoanalista no sabe, aunque el paciente le adjudica un lugar de saber (Lacan, 1983, p.116; 1990, pp.256-271). Tanto es así que el uso del diván permite al paciente desentenderse de la influencia que podría tener sobre él la expresión, el gesto no verbal del analista; así queda confrontado a sí mismo, con el soporte de un otro (representante del Otro) que lo escucha. Además, el concepto de sujeto supuesto saber alude también, desde la lectura que realizamos, al uso del poder en el dispositivo. Aunque el paciente confiere el poder al analista: "dígame qué me pasa, qué tengo que hacer....", este último se abstendrá del uso de su criterio, es decir del poder que se le confiere, en pos de la direccionalidad del deseo inconsciente de su paciente (Lacan, 1983). El encuadre, la materialidad y el espacio físico en el cual se desarrolla una sesión de psicoanálisis promueven el trabajo del sujeto en su relación transferencial. Precisamente es la transferencia, y su análisis 3 , la brújula de la cura del sujeto, aquella que, al mismo tiempo, define un trabajo clínico como psicoanalítico.

3"En la medida en que el analista hace callar en él el discurso intermedio para abrirse a la cadena de las verdaderas palabras, en esa medida puede colocar en ella su interpretación reveladora" (Lacan, 1983, p. 120). 
La modalidad de los dispositivos psicoanalíticos que se propone revisar, plantea de otro modo estos elementos: su disposición y operatividad en el respeto a la direccionalidad del deseo inconsciente del sujeto se produce en escenarios distintos al de la sesión uno a uno (un hablante y un escuchante), y diferentes también al del escenario grupal. Algunos autores refieren este trabajo con el nombre de "psicoanálisis aplicado"4; otros lo han conceptualizado como "psicoanálisis extramuros"y por fin

\footnotetext{
${ }^{4}$ Hay diferentes maneras de conceptualizar el Psicoanálisis Aplicado. Ciertos autores (Besse, 2011) lo proponen en el lindero de articulación con otras ciencias, disciplinas, saberes, o producciones, asumiendo el término mismo empleado por Freud en sus ensayos de psicoanálisis aplicado (Cornide, 2012) al analizar a través de conceptos psicoanalíticos obras literarias, producciones culturales o biografías. Sin embargo, hay otros autores que recuperan el término acuñado por Lacan (1964), Psicoanálisis aplicado, o psicoanálisis aplicado a la terapéutica, cuando se trata de un trabajo clínico, inicialmente planteado para un quehacer en el ámbito médico pero que en la actualidad trasciende el mismo (Maya, 2009). Miller propone que "se trata de psicoanálisis en su aplicación a cuestiones terapéuticas ( ...) se trata de aplicar todos los recursos del psicoanálisis, a diferencia de terapias hechas de modo más cómodo, que usan un método "simplificado" o degradado del psicoanálisis (Miller, J., D' Angelo, L., Fuentes, A., Garrido, C., Goya, A., Rueda, F., Vicens, A.,-2006, p. 14-). Trabajo psicoanalítico que no pretende el pase y el atravesamiento del fantasma, pero que al mismo tiempo se diferencia de la psicoterapia. El concepto de Psicoanálisis aplicado se diferencia también de aquello que suele denominarse Psicoanálisis en extensión (Besse, 2011).
}

algunos, como Doltó, no se molestaron en asignarle un nombre más que aquel que le pusieron quienes hicieron uso de él: la Casa Verde.

Sin embargo, al parecer, y según hemos podido apreciar en la clínica, es la ética psicoanalítica la que instaura una dinámica en la que los elementos en juego funcionan de modo parecido al anteriormente descrito para el dispositivo clásico, de otra manera y bajo otras condiciones; $y$, si bien en estas otras modalidades no se pretende una cura, se procura sí el advenimiento del sujeto. Así, es distinta la devolución del saber para el sujeto que lo produce y hay otra forma de abstención del poder por parte de quien devuelve ese saber. Así mismo, las características de las condiciones, o lo que en el dispositivo tradicional suele denominarse encuadre, cambian; pero, al igual que en la sesión analítica, el entorno físico y material en el cual se desarrolla el encuentro adquiere la función de una herramienta de trabajo. Aludiremos a estos elementos a propósito de dos dispositivos. 


\section{LA CASA VERDE: UN DISPOSITIVO COMUNITARIO PARA ACOGER LA PALABRA DE LOS PEQUEÑOS CON SUS PADRES}

Esta modalidad de intervención fue propuesta por Francoiçe Doltó con la intención de acoger las dificultades y malestares de la relación padres-hijos y cuya atención se consagra sobre todo hacia los procesos de separación en pos de la autonomía humanizada del pequeño, considerado en tanto sujeto a parte entera (Donzino, 2014; Doltó, 2004; Giani, M. y Fussimi, 2009; Parra, 2011, 2014; Sacks, 1997). Esa modalidad implementa una circulación del saber, y por tanto del poder, distintas a las del dispositivo psicoanalítico convencional. El dispositivo está planteado para permitir y favorecer procesos de simbolización o, como se ha hecho común, llamar apalabramiento. Para empezar, en estos lugares, aunque el espacio está concebido desde la perspectiva psicoanalítica, nadie ejerce una función de psicoanalista. El encuentro se organiza a partir de la función de acogimiento que es desempeñada por al menos dos personas que rotan día a día (un equipo de unas seis personas acuden de dos en dos) y de las cuales, al menos una, debe ser atravesada por el psicoanálisis o ser psicoanalista. Entre los conceptos importantes que operativizan el espacio se encuentra el de atención distraída. Se trata de una atención concomitante a la atención flotante de la cura tipo, y que otorga al espacio de un ambiente fluido y distendido que permite, al mismo tiempo, encontrar el momento más propicio para realizar una intervención desde la palabra. ¿Qué se interviene? ¿Cómo se interviene? Muchas veces las intervenciones se producen en torno a situaciones de evidente conflicto o de malestar expresado por los niños que acuden al espacio, también ante las verbalizaciones y acciones de los padres. Estas intervenciones buscan apalabrar situaciones en las cuales el afecto amenaza desbordante o aparece la agresividad. Se trata de intervenciones ligeras, a decir de Doltó, muchas de las cuales, incluso, pasan desapercibidas.

En la cotidianidad de estos espacios, en los cuales los niños disponen de diferentes objetos: juguetes varios, un espacio para pintar y dibujar, un espacio para jugar con agua que suele ser el favorito, ninguna actividad es previamente planificada; nadie dice lo que hay que hacer o decir; cada quien se ocupa en aquello que le llama la atención o le convoca. Así se genera una situación, quizás concomitante, para cada sujeto, a la de la asociación libre del dispositivo de la cura. Los niños y sus padres acuden 
dentro de un margen de horarios propuestos (encuadre) sin obligatoriedad, y pueden encontrarse cada vez con otros niños y otros padres, sin que llegue a conformarse un grupo. Entonces, en medio de la producción asociativa lúdica y discursiva, el conflicto, el malestar, se expresan: eventos como la pelea que entablan un par de niños disputándose un juguete, o el conflicto de un niño o niña con su acompañante ante la propuesta de un cambio de actividad por uno de los dos, invitan a la intervención del acogiente, un tercero, cambiante, que vehiculiza una palabra que rompe la especularidad en la cual los implicados se encuentran. Doltó (2006) manifestaba que muchas veces las disputas por objetos de los niños pequeños no implican un deseo del objeto sino un problema identificatorio: querer estar en el lugar del otro, querer ser el otro, y es sobre este querer imposible que interviene la palabra del tercero acogiente. Cuando esta situación es nombrada de modo preciso, adviene la calma como por acto de magia 5 .

Se afirma que la circulación del poder y del saber se producen de modo diferente en este dispositivo porque no existe un lugar permanente asignado para que alguien encarne ese lugar. La

${ }^{5}$ Una explicación detallada sobre las condiciones y dinámica que se produce en este tipo de dispositivo se encuentra en Cecilia Vaca, 2001. función de acoger, al ser rotativa y plural, elimina la posibilidad de centralizar estas funciones en una sola persona; así se limita el ejercicio del poder y del saber. Estar entre al menos dos permite una diversificación de las intervenciones, como también permite que uno pueda, si es necesario, tachar al otro (Otro), cuando la operación de un sujeto lo requiera, en el sentido de destituirlo de un lugar de saber obturante para la producción del sujeto (Cfr. Di Ciaccia, 2014). Asimismo, el modo como el dispositivo está planteado permite que los participantes, en determinados momentos, puedan mostrar su posición frente al poder, enunciar su saber, o devenir un tercero en una determinada situación de orden especular. Todas estas posibilidades toman curso gracias a las condiciones creadas para la verbalización y gracias al análisis de una forma particular de transferencia. La transferencia al lugar. Se trata de un concepto de Doltó que articula los elementos puestos en juego en el dispositivo. La idea que el personal de acogimiento sea rotativo y plural permite también una pluralización de la transferencia, así hay padres que afirman que les gusta venir más el día en que acoge tal o cual persona $y$, sin embargo, aquello no les impide acudir otros días puesto que el lugar permanece. De modo que el planteamiento de la Casa Verde permite la actualización de los afectos en la relación con algunas 
personas, sostenida en un encuadre material que en lo demás está fijo: el lugar de encuentro es el mismo, los objetos materiales se encuentran dispuestos siempre de la misma manera. Este doble movimiento de pluralidad en la relación con los acogientes y de singularidad en la relación con el lugar adquiere el estatuto de transferencia porque se analiza, para buscar el modo de plantear y precisar las intervenciones, durante las reuniones del equipo.

Un aspecto interesante respecto al planteamiento de esta práctica es que permite la instauración de espacios de palabra y simbolización articulados a diferentes situaciones sociales que por lo general generan sufrimiento en los niños y sus familias, situaciones en las cuales la relación padres-hijos se encuentra comprometida. Por ejemplo, el proyecto Policcino, llevado a efecto para permitir el encuentro "apalabrado" entre padres privados de la libertad y sus hijos, constituye una muestra de la versatilidad que conlleva el dispositivo (Solcà, 2008). En Bélgica y en España se han instaurado espacios de encuentro padres-hijos que facilitan la simbolización de situaciones de separación debidas a procesos judiciales, sostenidas en el planteamiento doltoniano.

Sin duda, la dinámica que producen estos dispositivos permite formular varias preguntas, entre ellas una que cuestiona permanentemente a los equipos que trabajan en estos dispositivos: ¿Las intervenciones realizadas pueden ser consideradas como intervenciones en el orden del análisis?

Sin llegar aún a establecer un espacio como la Casa Verde en nuestro medio, la operatividad de la ética psicoanalítica de estos dispositivos ha permitido pensar y realizar intervenciones clínicas ligeras en situaciones específicas como el acompañamiento con la palabra a bebés separados de sus madres o padres, a niños pequeños en situaciones de institucionalización, y también generar espacios de encuentro y de palabra padres-hijos de modo puntual, permitiendo operaciones parecidas a las del dispositivo planteado por Doltó en situaciones de violencia intrafamiliar.

\section{LA PRÁCTICA ENTRE VARIOS: UN DISPOSITIVO INSTITUCIONAL PARA ACOGER AL SUJETO}

El problema es que en la locura el loco no es el dueño de su cuerpo [...] Su libertad tiene el ámbito de los muros del manicomio o de los miserables cuartuchos de hotel a donde se los recluye hoy en día 
después de impregnarlos con productos químicos. [...] Entre la propuesta del amo que encierra y reduce al loco y el recurso idealista a una libertad insondable y fantasmática, el desafío para los psicoanalistas es el de encontrar una tercera vía. El determinismo freudiano y la causalidad estructural lacaniana indican la dirección a seguir. [...] El conjunto de la aventura psicótica resulta de esta dispersión de los significantes que han quedado invertebrados, desligados del vínculo social. La metáfora delirante intenta remendar la falla de la metáfora paterna en su función de conferir una significación a la falta en el Otro. Pretende devolver al sujeto a las redes del lazo social (Braunstein, 2006, pp. 270-278).

El desafío implicado en el trabajo con niños psicóticos llevó a un equipo de profesionales posicionados en el psicoanálisis lacaniano a implementar una modalidad de práctica en la cual las personas: profesionales, asistentes, empleados, en contacto con el niño en un centro especializado para situaciones de psicosis o perturbaciones graves, intervienen como compañeros de la operación subjetiva del mismo. Esta intervención requiere que quienes actúan, es decir cualquier persona en contacto con el niño afín a este modo de intervención, pueda actuar en el momento propicio, desde su propio estilo, aportando ya sea un significante que despliega y articula la red significante del paciente, ya sea un gesto significante que opera en el mismo sentido; o una destitución de su saber o su poder para apaciguar la omnipotencia del Otro no regulado de la psicosis (Di Ciaccia, 2014; Zenonni, 2006). Al parecer, los psicoanalistas de la práctica entre varios encontraron ese tercer planteamiento cuyo requerimiento es señalado por Braunstein. Una vía que devuelve al sujeto a su lazo social a través del soporte que le proporcionan sus otros. Nuevamente nos encontramos frente a la implementación de un dispositivo donde las funciones se dislocan, no hay un psicoanalista o al menos no requiere estar presente físicamente durante las intervenciones. Quien actúa resulta compañero -parternaire- de la operación subjetiva del niño, o paciente.

Hay literatura que recoge la riqueza clínica de las intervenciones o maniobras que realizan quienes se sienten avocados a ser compañeros de la operación subjetiva de un niño, niña o adulto en posición psicótica. Algunas de estas intervenciones incluso requieren el trabajo simultáneo de dos o más personas en el sentido de regular el goce mortífero del Otro del psicótico. 
La cercanía a esta práctica también ha permitido, en varias ocasiones, la puesta en marcha de intervenciones para el acompañamiento ambulatorio de personas psicóticas en nuestro medio, e incluso nos ha permitido pensar en la función de estas intervenciones en casos de neurosis.

Difícil en una síntesis general plasmar la riqueza, la creatividad y los efectos que despliegan estas intervenciones. Igualmente, en nuestro medio aún no hay instituciones que adscriban a esta práctica. Sin embargo, su existencia en otros contextos ha permitido el planteamiento de intervenciones puntuales en situaciones harto complicadas. Por mencionar una de las posibilidades que se abrieron a partir del planteamiento de la práctica entre varios, se puede mencionar cómo, en algunas situaciones específicas de acompañamiento ambulatorio a crisis agudas de pacientes psicóticos, la participación guiada de la autoridad, un juez, un teniente político, la policía comunitaria, alcanza a realizar aquella operación de regulación del Otro requerida por la persona psicótica.

Y, precisamente, este tipo de praxis se revela de interés cuando hemos atestiguado la brecha entre la política de salud, que instaura la necesidad que las personas con psicosis sean acogidas y tratadas en la comunidad, así como la falta de métodos y servicios de acom- pañamiento ambulatorio a la que se confrontan las familias de estos pacientes. Más allá del ideal abstracto de una permanencia y tratamiento en la comunidad, la práctica entre varios permite el planteamiento de un proceso de acompañamiento afincado en las posibilidades y condiciones que ofrece la realidad.

Así, en alguna ocasión, debido a la falta de camas psiquiátricas, en el momento del advenimiento de una crisis delirante se recurrió a la intervención guiada de la policía comunitaria: el patrullero del barrio paseó cada noche, durante varias semanas, por la calle de residencia de la paciente, para protegerla de la persecución que vivía en su delirio; al parecer, este acto contribuyó a que pudiera sostenerse en su cotidianidad sin internamiento.

Sorprendente la disposición que hemos encontrado tanto por parte de los ciudadanos comunes y corrientes como de los funcionarios de las instituciones para colaborar en este tipo de intervenciones cuando son informados de su lógica subyacente.

Los dispositivos mencionados u otros parecidos se han instaurado en algunos países latinoamericanos, generando reflexiones, conocimientos y nuevas articulaciones teóricas. En el contexto ecuatoriano, el planteamiento de una praxis psicoanalítica transdisciplinaria con la comunidad, que produzca un es- 
pacio para la escucha del inconsciente y la lógica del sujeto, se vislumbra con posibilidades. De hecho, como se ha mencionado, hay experiencias que han tenido lugar en unas pocas instituciones en las cuales algún profesional con filiación psicoanalítica instala esta posibilidad, la misma que suele estar circunscrita a pedidos terapéuticos o de atención psicológica en su intersección con el accionar de otras disciplinas. Se trata aún de una práctica poco convencional. Aledañamente, y en una perspectiva parecida, se han desarrollado en nuestro contexto dispositivos de orientación psicoanalítica en el ámbito social, específicamente en el ámbito de intervención con la población de niños y familias ligadas a situaciones de trabajo infantil (Egas, et al, 2013). Estos dispositivos implementan estrategias que contemplan la lógica del sujeto y que producen saberes desde los propios usuarios y actores implicados.

Desde estas experiencias, poco difundidas, ha sido posible constatar que la colaboración para intervenciones clínicas que consideran la lógica del sujeto puede ser bien acogida y se realiza, por lo general, con buena disposición e incluso entusiasmo por parte de las personas invitadas a participar.

Ciertamente hay que afinar los modos de intervención, pulirlos, cuidando a los intervinientes y delimitando éticamente el estatuto de las interven- ciones, aspecto crucial que despierta múltiples interrogantes. También ha sido posible apreciar otras dificultades que este tipo de trabajo puede generar, dificultades del orden de lo especular en las relaciones que se suscitan, o intervenciones parecidas a la interpretación silvestre ya denunciada por Freud. Sin embargo, la disposición está presente y en ocasiones se devela de singular potencia y efectividad de corte. Se trata quizás de una praxis que evoca una antigua afinidad cultural hacia el trabajo colaborativo y la participación comunitaria.

Son varios los autores que han cuestionado fuertemente la dicotomía individuo-sociedad. Quizás este tipo de dispositivos permiten insertarnos en el corazón de este cuestionamiento. Pensar en dispositivos psicoanalíticos que se salen de la relación uno a uno (en su formalidad), nos lleva a problematizar y articular teóricamente la relación sujeto-comunidad-cultura. Algunas otras pistas pueden ser tomadas de la explicación milleriana sobre el cambio conceptual realizado por Lacan en su propuesta del seminario Aún, en el cual el término hablanteser sustituye al de sujeto en un intento de articular la dimensión pulsional y de goce al lenguaje: "El lazo social es el término que responde a la relación sexual [...] Estos dos términos deben ponerse en paralelo, y es necesario decir que cuanto más se avanza en lo que se 
llama la historia, más consistencia toma el ser comunitario" (Miller, 2008:167).

O quizás, retomando al propio Lacan:

"Esa palabra que constituye al sujeto en su verdad, le esta sin embargo vedada para siempre (...) Pero si esa palabra es no obstante accesible, es que ninguna verdadera palabra es únicamente palabra del sujeto, puesto que es siempre fundándola en la mediación de otro sujeto como ella opera, y puesto que por ese camino está abierta a la cadena sin fin -pero sin duda no indefinida, puesto que se cierra- de las palabras donde se realiza concretamente en la comunidad humana la dialéctica del reconocimiento" (1983, p.120)

Además, este aspecto lleva a pensar que posiblemente este tipo de intervenciones clínicas, sostenidas en la escucha y la teoría psicoanalítica, podrían parecer cercanas, a través de su planteamiento, a la psicoterapia comunitaria que se practica en varios países latinoamericanos. Pero al mismo tiempo es necesario enfatizar que guardan una especificidad que las diferencia: aquella de la implicación del sujeto del inconsciente. Se trata entonces de desbrozar, definir, delimitar las diferencias y los encuentros entre estos tipos de prácticas, interrogarse permanentemente sobre su dimensión clínica, y fundamentalmente sobre sus riesgos.

También queda el reto de circunscribir mejor, y de modo más preciso, el campo epistemológico en el cual se inscriben este tipo de prácticas así como discernir sus efectos en la subjetividad. Curiosamente, cuando se habla del trabajo psicoanalítico en el campo de la infancia, suele aparecer una máxima que se ha extendido. Se dice: Psicoanálisis con niños, no Psicoanálisis de niños, porque el modo de trabajar con los niños se sale ya del dispositivo de la cura-tipo. Es decir que, para algunos psicoanalistas 6 , el trabajo psicoanalítico con niños constituiría ya una modalidad de psicoanálisis aplicado. Revisar estos dispositivos poco convencionales que requieren la intervención de algunos otros, nos lleva a una pregunta parecida y a pensar que, si ciertamente no podemos poner a una comunidad en el diván, cuando los miembros de una comunidad se vuelven compañeros y habilitantes de la operación subjetiva de uno de sus conciudadanos quizás no resulta tan errado decir: Psicoanálisis con la comunidad.

${ }^{6}$ Esta no es sin embargo una posición unánime, por ejemplo para Jerusalinsky se trata de Psicoanálisis de niños (Jerusalinsky, 2002, p. 17). 
ReVista PUCE, ISSN 1390-7719. Núm.103. 3 de mayo de 2016 -

\section{Bibliografía}

Allouch, J. (1994). Freud, Y Después Lacan. Buenos Aires Capital Federal: Edelp

Anzieu y Martin (1971). La dinámica de los grupos pequeños. Buenos Aires: Kapelusz. Recuperado de:

http://www.teoriaymetodologiats.ecaths.com/ver-trabajos-practicos/33550/el-concepto-de-grupo-

anzieu/file:///D:/HP/

Downloads/1256615331.anzieu\%20 martin\%20la\%20dinamica\%20de\%20 lo\%20peque\%C3\%B1 os\%20grupos.pdf. 20-06-2015

Besse, J. (2011). El porvenir de un Encuentro. Psicoanálisis y Ciencias Sociales. Entre la Aplicación y la Extensión. En Escolar, C. y Besse, J. (2011) Epistemología fronteriza: puntuaciones sobre teoría, método y técnica en ciencias sociales (1a. ed.). Argentina: Eudeba, 2011. ProQuest ebrary. $28-03-2016$.

Braunstein, N. (2006). El Goce. Un concepto lacaniano. Buenos Aires: Siglo XXI.

Braunstein, N. (2003). Psicología Ideología y Ciencia. México D.F.: Siglo XXI

Buzzaqui, A (1998). El grupo operativo de Enrique Pichón Riviére. Análisis y Crítica. Tesis Doctoral Universidad Complutense de Madrid, Facultad de ciencias Políticas y Sociología. Departamento de Psicología Social. Recuperado de: http://biblioteca.ucm.es/tesis/cps/ ucm-t23006.pdf, 25-05-2015
Cornide, E. (2012) El Psicoanálisis Aplicado. Un camino de ida y vuelta. Buenos Aires. Asociación Psicoanalítica de Buenos Aires. Recuperado de: www. apdeba.org/wp-content/.../EL-PSICOANÁLISIS-APLICADO-CORNIDE.doC 28-032016.

Di Ciaccia, A. (2014). La Practique á Plusieurs. Publicado en: Errancia. La palabra inconclusa. Revista de Psicoanálisis, teoría crítica y cultura. № 8, 2014. Recuperado de: http://www.iztacala.unam. mx/errancia/v8/PDFS_1/TEXTO\%20LITORALES\%201\%20ERRANCIA\%208.pdf, 25-05-2015.

Doltó F. (2006). En el juego del deseo. México: Siglo XXI.

Doltó, F. (2004). La causa de los niños. Barcelona: Paidós.

Donzino, G. (2014). Aída Chernicoff de Saks in memorian. Recuperado de: dspace.uces.edu.ar:8180/xmlui/ bitstream/handle/.../Aida_Donzino.pdf

Egas, et al. (2013). Psicólogos fuera de los Muros de la Consulta. Experiencia de orientación psicoanalítica dentro del programa de erradicación de trabajo infantil-Proniño. Quito: Universidad SEK.

Freud, S. (1992) Psicología de las masas y análisis del yo (1921) en Obras Completas Vol. 18. Buenos Aires: Amorrortu.

Giani, M. y Fussimi, C. (2009). Herramientas subjetivas que protegen. Una propuesta de incorporación de prácticas de salud mental en los procesos de aten- 
ción de niños y niñas pequeños. Publicado en: Neuquem tierra de confluencias. Neuquem: Gobierno de la Provincia de Neuquem. Recuperado de: http://www4. neuquen.gov.ar/salud/images/archivo/ Programas_prov/Guia_de_Atencin_y_ Cuidado_del_menos_de_6_anos/Herramientas_subjetivas_que_protegen_ ago_2011.pdf 07-12-2012.

Gordon, W. Bain y A. Gould, L. (1996). The fifth basic assumption. Publicado en Free Associations. Volume 6, Parte 1 ( $\left.N^{\circ} 37\right): 2855$. Recuperado de: http:// www.acsa.net.au/articles/thefifthbasicassumption.pdf. 20-06-2015.

Jerusalinsky, A. (2002) Para entender al niño. Claves Psicoanalíticas. Quito: Abya-Yala

Käes, R. (1997) El estatuto teórico clínico del grupo. De la psicología social al psicoanálisis. Publicado en: Tramas No11. México: UAM-X, 221-257.

Lacan, J. (1947) "La psiquiatría inglesa y la guerra". Recuperado de http:// www.elseminario.com.ar/biblioteca/Lacan La psiquiatria inglesa y la guerra. pdf. 27-03-2015.

Lacan, J. (1990). Les quatre concepts fondamentaux de la psychanalyse. Paris: Seuil.

Lacan, J. (1983) "Variantes de la Cura Tipo" en Escritos II 8va edición. México: Siglo XXI

Lacan, J. (1964) "Acto de Fundación 21 de Junio de 1964" Escuela Freu- diana de Paris. Recuperado de: http:// www.eol.org.ar/template.asp? Sec $=1 a_{\text {_ }}$ escuela\&SubSec=estatutos\&File=estatutos/acto_fundacion.html.27-03-2015

Laurent, E. (2000). Psicoanálisis y Salud Mental. Buenos Aires: Tres Haches.

Mannoni, M. (1992). Un saber que no se sabe. La experiencia analítica. BarceIona: Gedisa.

Maya (2009). Tres vías un método. En revista Affectio Societatis, No 11, Diciembre. Pp. Recuperado de: http://antares.udea.edu.co/ psicoan/affectio11. html. 27-03-2016

Miller, J., D' Angelo, L., Fuentes, A., Garrido, C., Goya, A., Rueda, F., Vicens, A. (2006). Efectos terapéuticos rápidos Conversaciones Clínicas con Jacques-Alain Miller en Barcelona. Instituto del Campo freudiano. Sección clínica de Barcelona. Buenos Aires: Paidós

Miller, J. (2008). El partenaire-síntoma. Los cursos psicoanalíticos de Jaques -Alain Miller. Buenos Aires: Paidós.

Parra, M. (2014). Dispositivos Colectivos en Salud Mental e Intervenciones Clínicas con niños pequeños y sus familias. VI Congreso Internacional de Investigación y Práctica Profesional en Psicología XXI Jornadas de Investigación Décimo Encuentro de Investigadores en Psicología del MERCOSUR. Facultad de Psicología - Universidad de Buenos Aires, Buenos Aires. Recuperado de: http://www. aacademica.com/000-035/517 
Revista PUCE. ISSN 1390-7719. Núm.103. 3 de mayo de 2016 3 de noviembre de 2016. Cecilia VACA H. PP, 127-147 \#\|l\|

Parra, M. (2011). ¿Qué es casa "arco iris"? Caracterización de un dispositivo de salud mental comunitaria para niños pequeños y sus familias". En Revista Calidad de Vida UFLO - Universidad de Flores Año III, Número 6, V1, pp. 138-157. 1850-6216 URL del Documento: cienciared.com.ar/ra/doc.php?n=1537 URL de la Revista: cienciared.com.ar/ra/revista. php? wid=4

Sacks, A. (1997). Nueva Estrategia en la Prevención de la violencia "Casa verde de los niños". Recuperado de:

http://dspace.uces.edu. ar:8180/xmlui/bitstream/handle/123456789/440/Casa_verde_de_los_ ni\%C3\%B1os.pdf? sequence $=3$

Solcà (2008). Jornadas internacionales de espacios familiares de la pequeña infancia, 2008. Barcelona. Disponible en elcuartodeestar.files.wordpress.com/.../ ponencias-de-las-jornadas-interna.

Vaca, C. (2001). La Construcción de Dispositivos en la Pluralidad. Aproximación a la Clínica en un Centro de Salud Mental. Memoria- Informe de Prácticas realizado para la obtención de un DES en Ciencias Psicológicas. Universidad Católica de Lovaina. Bélgica.

Zenoni, A. (2006). Orientación analítica en la institución psiquiátrica. Publicado en: Bitácora lacaniana el psicoanálisis hoy. $N^{\circ} 1$, mayo de 2006. Recuperado de: http://contrasentido.net/ wp-content/uploads/2007/01/a zeno- ni-orientacion-analitica-en-la-institucion-psiquiatrica.pdf,25-05-2015 
